\title{
Cinética de inativação da peroxidase em água de coco
}

\section{Inactivation kinetics of peroxidase from coconut water}

\author{
Rafael da Costa Ilhéu Fontan ${ }^{1 *}$; Lizzy Ayra Pereira Alcântara ${ }^{2}$; Saint Clair Assis \\ Laje Neto $^{3}$; Renata Cristina Ferreira Bonomo ${ }^{4}$; Gabrielle Cardoso Reis Fontan ${ }^{5}$
}

\section{Resumo}

Este trabalho teve como objetivo estudar a cinética de inativação térmica da enzima peroxidase (POD) presente na água de coco verde, determinando-se os valores dos parâmetros $D, z$ e $Q_{10}$, além da energia de ativação $\left(E_{a}\right)$ de Arrhenius para o processo. Foram avaliados nove binômios tempo/temperatura para o tratamento térmico em três temperaturas $\left(75^{\circ} \mathrm{C}, 85^{\circ} \mathrm{C}\right.$ e $\left.95^{\circ} \mathrm{C}\right)$ e tempos de manutenção variando de $1 \mathrm{~min}$ a $30 \mathrm{~min}$, além da amostra sem tratamento térmico. Os valores de $D$ foram determinados por meio de regressão linear, obtendo-se os valores de 833,3min., 35,6min. e 1,63min., para as temperaturas de $75^{\circ} \mathrm{C}, 85^{\circ} \mathrm{C}$ e $95^{\circ} \mathrm{C}$, respectivamente, enquanto o valor de $z$ foi igual a $7,4^{\circ} \mathrm{C}$. Esses valores demonstraram que a POD da água de coco é resistente à inativação térmica, sendo tal processo fortemente afetado pela temperatura utilizada. Tal fato foi reforçado pelos altos valores da $E_{a}(332,3 \mathrm{~kJ} / \mathrm{mol})$ e $Q_{10}(22,7)$, que indicaram que uma alta quantidade de energia é requerida para a inativação da POD e que o aumento da temperatura aumenta a velocidade de inativação.

Palavras-chave: Valor $D$, valor z, energia de ativação, $Q_{10}$, atividade enzimática

\begin{abstract}
The aim of this project was study the thermal inactivation kinetics of the peroxidase enzyme (POD) present in the green coconut water, being determined the parameters $D$ values, $z$ value and $Q_{10}$, besides the Arrhenius' activation energy $\left(E_{a}\right)$ to the process. Nine time/temperature binomials for the thermal treatment at three temperatures $\left(75^{\circ} \mathrm{C}, 85^{\circ} \mathrm{C}\right.$ and $\left.95^{\circ} \mathrm{C}\right)$ and maintenance times from $1 \mathrm{~min}$ to $30 \mathrm{~min}$, besides a control sample without thermal treatment were evaluated. $D$ values were obtained by linear regression, being equals to $833,3 \mathrm{~min}, 35,6 \mathrm{~min}$ and $1,63 \mathrm{~min}$ at $75^{\circ} \mathrm{C}, 85^{\circ} \mathrm{C}$ and $95^{\circ} \mathrm{C}$, respectively, while the $z$ value was equal to $7,4^{\circ} \mathrm{C}$. These values demonstrate that POD of coconut water is thermal resistant to inactivation, being such processes strongly affected by temperature. Such fact is also verified by the high values of $E_{a}(332,3 \mathrm{~kJ} / \mathrm{mol})$ and $Q_{10}(22,7)$, which indicate that a high amount of energy is required for the POD inactivation and an increase of temperature elevate the inactivation rate.
\end{abstract}

Key words: $D$ value, $z$ value, activation energy, $Q_{10}$, enzyme activity

1 Prof. Assistente, Dept $^{\circ}$ de Tecnologia Rural e Animal, DTRA, Universidade Estadual do Sudoeste da Bahia, UESB, Itapetinga, BA. E-mail: rafaelfontan@yahoo.com.br

2 Pós-Graduanda em Ciência e Tecnologia de Alimentos, Dept ${ }^{\circ}$ de Tecnologia de Alimentos, Universidade Federal de Viçosa, UFV, Viçosa, MG. E-mail: lizzy.alcantara@gmail.com

3 Engenheiro de Alimentos, DTRA, UESB, Itapetinga, BA. E-mail: saint.engalim@hotmail.com

4 Pesquisadora Nível 2 do CNPq, Prof Titular, DTRA, UESB, Itapetinga, BA. E-mail: bonomorcf@pq.cnpq.br

5 Prof ${ }^{\mathrm{a}}$ Assistente, DTRA, UESB, Itapetinga, BA. E-mail: gcardosoreis@yahoo.com.br

* Autor para correspondência 


\section{Introdução}

A água de coco verde (Cocos nucifera L.) é o líquido do endosperma encontrado dentro da cavidade do coco, que começa a se formar em torno de dois meses após a abertura natural da inflorescência. Apresenta sabor doce e levemente adstringente e valores de $\mathrm{pH}$ na faixa de 4,0 a 5,6, conforme a variedade e grau de maturação. Em sua composição, a água de coco apresenta aproximadamente $95 \%$ de água, $4 \%$ de carboidratos, $0,1 \%$ de gordura, $0,02 \%$ de cálcio, $0,01 \%$ de fósforo, $0,5 \%$ de ferro, além de aminoácidos, vitamina $\mathrm{C}$, vitaminas do complexo $\mathrm{B}$ e sais minerais (CAMPOS et al., 1996; CARVALHO et al., 2006).

É um produto perecível e tradicionalmente comercializado dentro do próprio fruto, o que envolve problemas relativos ao transporte e armazenamento. Algumas técnicas de processamento vêm sendo empregadas pelas indústrias para permitir o seu envase, proporcionando um aumento da vida útil e conservando suas características sensoriais originais (MAGALHÃES et al., 2005).

A atividade enzimática na água de coco é um fator de grande relevância para sua comercialização devido às alterações indesejáveis que acarreta, mesmo quando conservada de forma adequada. Desta forma, determinar as condições em que ocorra a inativação enzimática implica em obter um produto seguro e de qualidade sensorial (CAMPOS et al., 1996; MAGALHÃES et al., 2005).

Diversos autores observaram a presença de polifenoloxidases (PPO) e peroxidases (POD) na água de coco verde, sendo estas as responsáveis pela mudança de coloração da água de coco após sua extração do fruto (CAMPOS et al., 1996; DUARTE; COELHO; LEITE, 2002; MATSUI et al., 2007), além de estarem intimamente associadas à deterioração de diversos nutrientes como o ácido ascórbico (ROBINSON; ESKIN, 1991).

Peroxidases (POD) são um grupo de enzimas que catalisam reações de oxidação, reduzindo o peróxido de hidrogênio a água, enquanto oxida uma variedade de substratos. Esta enzima apresenta o máximo de atividade em pH 5,5 e temperatura de 35 ${ }^{\circ} \mathrm{C}$, é resistente ao calor, sendo considerada indicador biológico de tratamento térmico (ROBINSON; ESKIN, 1991; FELLOWS, 2006). O estudo dessa enzima proveniente de diversas fontes botânicas vem sendo realizada por diversos pesquisadores, buscando entender a sua cinética e mecanismo de ação (ZANATTA; ZOTARELLI; CLEMENTE, 2006; LUÍZ; HIRATA; CLEMENTE, 2007; FREITAS et al., 2008; GALDINO; CLEMENTE, 2008).

Campos et al. (1996) relataram que o tratamento térmico para inativação da peroxidase na água de coco verde só é eficiente e completo acima de $90^{\circ} \mathrm{C}$ após 300 segundos de exposição do produto a esta temperatura, mas a partir de 90 segundos de exposição, já ocorrem problemas sensoriais relacionados às mudanças no aroma e sabor da água de coco.

A redução do $\mathrm{pH}$ não tem sido apresentada como um método eficiente de inativação enzimática por si só, uma vez que a água de coco apresenta características tamponantes. Verificou-se também que a refrigeração não é suficiente para a inibição completa da atividade enzimática na água de coco. À temperatura de $5^{\circ} \mathrm{C}$, o percentual de atividade enzimática é de 39\% em relação à temperatura ótima para a peroxidase (CAMPOS et al., 1996).

No estudo da cinética de inativação térmica de enzimas faz-se necessário o conhecimento de alguns parâmetros, que tornam possível a determinação do melhor binômio tempo/temperatura. $\mathrm{O}$ valor $D$ ou tempo de redução decimalé definido como o intervalo de tempo à temperatura de tratamento constante para uma redução de $90 \%$ da atividade enzimática, inicialmente presente no produto. $\mathrm{O}$ indicador da sensibilidade térmica do valor $D$ é definido como $z$, que é a diferença de temperatura requerida para reduzir o valor de $D$ em $90 \%$. O coeficiente de temperatura $\left(Q_{10}\right)$ por sua vez denota o quanto mais rápido uma reação (no caso a inativação enzimática) 
ocorre quando a temperatura é elevada de $10^{\circ} \mathrm{C}$. O bom entendimento do significado destes parâmetros aliado à correta manipulação matemática permite prever o comportamento da cinética de inativação para condições não executadas experimentalmente, ou seja, a partir dos dados de alguns ensaios, é possível predizer resultados em sistemas reais não estudados (PENNA; MACHOSHVILI, 1997; ELLOLY; AWAD; MANSOUR, 2007).

Assim, o objetivo neste trabalho foi estudar a cinética de inativação térmica da enzima peroxidase presente na água de coco verde, determinando os valores de $D$ para as temperaturas de $75^{\circ} \mathrm{C}, 85^{\circ} \mathrm{C}$ e $95^{\circ} \mathrm{C}$ e o valor de $z, Q_{10}$ e energia de ativação na faixa estudada.

\section{Material e Métodos}

\section{Material}

Foram adquiridos no comércio local da cidade de Itapetinga - BA, cocos verdes da variedade anão, colhidos entre o sexto e sétimo mês após a infloração dos coqueiros, não apresentando manchas nem injúrias mecânicas. Ao todos foram utilizados quinze frutos, divididos em três lotes de cinco unidades, a partir dos quais foi extraída a água de coco de forma manual. Na sequência a água de coco foi filtrada e acondicionada em recipiente plástico com tampa e mantido sob refrigeração $\left(5^{\circ} \mathrm{C}\right)$ até seu uso, o que ocorria no mesmo dia.

Para a determinação da atividade enzimática da peroxidase, foram utilizados os reagentes químicos Fosfato de Sódio Monobásico $\left(\mathrm{NaH}_{2} \mathrm{PO}_{4}, \mathrm{MM} 119,98\right.$ $\left.\mathrm{g} \times \mathrm{mol}^{-1}\right)$, Fosfato de Sódio Bibásico $\left(\mathrm{Na}_{2} \mathrm{HPO}_{4}\right.$, MM141,96g $\left.\times \mathrm{mol}^{-1}\right)$, Peróxido de Hidrogênio $\left(\mathrm{H}_{2} \mathrm{O}_{2}\right.$, MM 34g $\left.\times \mathrm{mol}^{-1}\right)$ e Guaiacol $\left(\mathrm{C}_{7} \mathrm{H}_{8} \mathrm{O}_{2}, \mathrm{MM} 124,137\right.$ $\left.\mathrm{g} \times \mathrm{mol}^{-1}\right)$, todos de grau analítico.

\section{Planejamento experimental}

Para se estudar a cinética de inibição da peroxidase, foi planejado um experimento no delineamento inteiramente casualizado com nove binômios tempo/temperatura diferentes: três deles a $75^{\circ} \mathrm{C}(10,20$ e $30 \mathrm{~min})$, três a $85^{\circ} \mathrm{C}(5,10$ e $15 \mathrm{~min})$ e três a $95^{\circ} \mathrm{C}(1,2$ e $3 \mathrm{~min})$, selecionados a partir de ensaios preliminares, além da amostra controle a qual não foi submetida a qualquer tratamento térmico. O experimento foi realizado com três repetições, totalizando 30 unidades experimentais. Análise de regressão linear foi realizada para a determinação dos parâmetros cinéticos, sendo a adequação dos modelos avaliada com base na falta de ajuste dos mesmos $(\mathrm{P}>0,05)$, significância dos parâmetros $(\mathrm{P}<0,05)$ e coeficiente de determinação $\left(\mathrm{R}^{2}\right)$ corrigido pela soma de quadrados de tratamentos.

\section{Tratamento térmico das amostras}

Para a realização dos tratamentos térmicos nos binômios tempo/temperatura pré-determinados, foram adicionados $5 \mathrm{~mL}$ de amostra de água de coco em tubos de ensaio. Em seguida, os tubos foram colocados em um banho termostático (Quimis, Modelo Q214S2, Diadema-SP, Brasil, precisão de $\pm 0,1{ }^{\circ} \mathrm{C}$ ) para o aquecimento nas temperaturas de interesse. As amostras foram aquecidas entre $75^{\circ} \mathrm{C}$ e $95^{\circ} \mathrm{C}$ para diferentes períodos de tempos. O tempo e temperatura foram monitorados com o auxílio de um cronômetro e um termômetro, respectivamente. Posteriormente, as amostras foram rapidamente resfriadas e mantidas em banho de gelo até a determinação da atividade enzimática da peroxidase.

\section{Determinação de atividade enzimática da peroxidase (POD)}

$\mathrm{Na}$ determinação da atividade enzimática da POD, foi utilizada uma adaptação da metodologia de Campos et al. (1996). A atividade da peroxidase foi determinada adicionando-se a um tubo de ensaio $2,3 \mathrm{~mL}$ de solução-tampão de fosfato de sódio $0,02 \mathrm{M}$ e pH 5,5; $0,2 \mathrm{~mL}$ de uma solução de peróxido de hidrogênio $0,1 \% \mathrm{v} / \mathrm{v}$ e $0,5 \mathrm{~mL}$ de uma 
solução alcoólica de guaiacol $0,5 \% \mathrm{v} / \mathrm{v}$, levandose ao banho de água até atingir a temperatura de $35^{\circ} \mathrm{C}$. Adicionou-se $1,0 \mathrm{~mL}$ da amostra de água de coco, procedendo-se imediatamente a leitura em espectrofotômetro (Femto, Modelo 700plus, São Paulo-SP, Brasil), no comprimento de onda de $460 \mathrm{~nm}$. A mistura foi retornada ao banho de água a $35^{\circ} \mathrm{C}$ por mais $10 \mathrm{~min}$, quando então uma nova leitura foi realizada. Utilizou-se como branco a mistura de todos os reagentes, substituindo-se $1,0 \mathrm{~mL}$ de amostra por $1,0 \mathrm{~mL}$ de água destilada.

A atividade foi expressa em unidades $/ \mathrm{mL}$ por minuto. Uma unidade equivale a uma variação de 0,001 na absorvância por minuto por $\mathrm{mL}$ de amostra. Para o cálculo de atividade da enzima, utilizou-se a seguinte equação:

$$
\operatorname{Ativ}\left(U \cdot m L^{-1}\right)=\frac{(A F-A I)_{\text {amostra }}-(A F-A I)_{\text {branco }}}{0,001 \cdot t}
$$

Em que, $A F$ é a absorvância final, $A I$ é a absorvância inicial e $t$ é o tempo em minutos.

Determinação dos parâmetros cinéticos $D, z, E_{a} e$ $Q_{10}$

A inativação térmica da POD segue o comportamento de reação cinética de reação de primeira ordem, possuindo um período inicial de inativação rápida seguido por um período de inativação branda (LING; LUND, 1978).

De acordo com o modelo de Bigelow (BALL; OLSON, 1957), o valor D ou tempo de redução decimal corresponde ao recíproco da inclinação da reta obtida plotando-se o logaritmo da atividade residual da POD em função do tempo. Assim, os valores de $\mathrm{D}$ foram obtidos por meio de uma regressão linear, conforme o modelo apresentado na equação 2:

$$
\log \left(\frac{A t i v}{A t i v_{0}}\right)=\frac{1}{D} \cdot t
$$

Em que Ativ $_{0}$ é a atividade inicial da enzima (no tempo $\mathrm{t}=0$ ) e Ativ é a atividade da enzima após o tratamento térmico durante um período de tempo $t$.

Uma vez que o valor $\mathrm{D}$ é dependente da temperatura do tratamento térmico, é necessário calcular seu valor para cada temperatura estudada. A variação experimental do valor D com a temperatura é uma relação exponencial, linearizada em um gráfico do logaritmo do valor D versus temperatura. Esta curva reflete a resistência relativa da população em estudo a diferentes temperaturas. $\mathrm{O}$ valor $\mathrm{z}$, ou constante de resistência térmica, corresponde ao aumento necessário na temperatura para que o valor D sofra uma redução de um ciclo logarítmico, sendo matematicamente igual ao recíproco da inclinação da reta no gráfico acima citado. Assim, o valor z para a peroxidase foi calculado de acordo com equação 3:

$$
\log (D)=\frac{1}{z} \cdot T+B
$$

$\mathrm{O}$ coeficiente de temperatura, $\mathrm{Q}_{10}$, para aumento da velocidade de inativação enzimática foi calculado como o valor médio para os valores de Q10 nos intervalos de temperatura de $75^{\circ} \mathrm{C}$ a $85^{\circ} \mathrm{C}$ e de $85^{\circ} \mathrm{C}$ a $95^{\circ} \mathrm{C}$, sendo utilizada a seguinte equação:

$$
Q_{10}=\exp \left[-\frac{E_{a}}{R}\left(\frac{1}{T+10}-\frac{1}{T}\right)\right]
$$

Em que $E_{a}$ é a energia de ativação $\left(\mathrm{J} \times \mathrm{mol}^{-1}\right)$ da equação de Arrhenius, $R$ é a constante universal dos gases ideais $\left(\mathrm{J} \times \mathrm{mol}^{-1} \times \mathrm{K}^{-1}\right)$ e $T$ é a temperatura $(\mathrm{K})$ inferior da faixa avaliada.

A energia de ativação para a inativação enzimática foi obtida do coeficiente angular da forma linearizada da equação de Arrhenius (equação 5) no 
gráfico de $\ln (\mathrm{k})$ versus $1 / \mathrm{T}$, onde $\mathrm{k}$ é a constante de velocidade da inativação enzimática a uma dada temperatura T (em Kelvin).

$$
\ln (k)=-\frac{E_{a}}{R} \cdot \frac{1}{T}+\ln \left(k_{0}\right)
$$

Assumindo a taxa de redução da atividade enzimática como uma reação de primeira ordem, podemos relacionar o valor de $\mathrm{k}$ com o valor $\mathrm{D}$ usando-se a equação 6 .

$$
k=\frac{1}{D \cdot \log (e)}
$$

\section{Resultados e Discussão}

As amostras de água de coco foram submetidas ao tratamento térmico em diversas condições de temperatura e tempo. Na Tabela 1 estão apresentados os valores de atividade residual da POD para cada binômio temperatura/tempo.

Tabela 1. Atividade enzimática da POD da água de coco em função da temperatura e do tempo de tratamento térmico.

\begin{tabular}{cccccc}
\hline & $\mathbf{T}=\mathbf{7 5}^{\circ} \mathbf{C}$ & \multicolumn{2}{c}{$\mathbf{T}=\mathbf{8 5}^{\circ} \mathbf{C}$} & \multicolumn{2}{c}{$\mathbf{T}=\mathbf{9 5}^{\circ} \mathbf{C}$} \\
\hline $\begin{array}{c}\mathbf{t} \\
(\mathbf{m i n})\end{array}$ & $\begin{array}{c}\text { Ativ. } \\
(\mathbf{U} / \mathbf{m L})\end{array}$ & $\begin{array}{c}\mathbf{t} \\
(\mathbf{m i n})\end{array}$ & $\begin{array}{c}\text { Ativ. } \\
(\mathbf{U} / \mathbf{m L})\end{array}$ & $\begin{array}{c}\mathbf{t} \\
(\mathbf{m i n})\end{array}$ & $\begin{array}{c}\text { Ativ. } \\
(\mathbf{U} / \mathbf{m L})\end{array}$ \\
\hline 0 & 30,23 & 0 & 30,23 & 0 & 30,23 \\
10 & 28,76 & 5 & 24,12 & 1 & 20,00 \\
20 & 28,68 & 10 & 17,11 & 2 & 2,70 \\
30 & 27,64 & 15 & 11,51 & 3 & 0,53 \\
\hline
\end{tabular}

$\mathrm{t}=$ tempo

Ativ. = atividade enzimática.

Na Tabela 1, observa-se que após 30 minutos de tratamento térmico a $75^{\circ} \mathrm{C}$, a atividade residual da POD foi bastante alta, aproximadamente 91\% da atividade enzimática inicial, o que indica que o tratamento térmico a essa temperatura proporciona reduzida inativação da $\mathrm{POD}$, necessitando de muito tempo (mais de $800 \mathrm{~min}$.) para que o tratamento seja eficiente (pelo menos $90 \%$ de redução da atividade). Já para o tratamento a $85^{\circ} \mathrm{C}$ observou-se um decréscimo mais acentuado da atividade enzimática da POD com o aumento do tempo de tratamento térmico, sendo que após 15 minutos de tratamento térmico, a atividade residual da POD nas amostras de água de coco foi de $38 \%$ em relação a atividade enzimática inicial.

Já para o tratamento a $95^{\circ} \mathrm{C}$ observou-se redução na atividade enzimática da POD com incrementos de tempo no tratamento térmico quando comparado às demais temperaturas em estudo. Após três minutos de tratamento a atividade residual da POD foi de apenas $1,8 \%$ da atividade enzimática inicial.

Os resultados da inativação térmica da POD estão de acordo com o que foi reportado na literatura, onde alguns autores verificaram que o tratamento térmico para inativação da enzima peroxidase na água de coco verde, só é eficiente acima de $90^{\circ} \mathrm{C}$ (CAMPOS et al., 1996).

Tempo de redução decimal (valor D) a diferentes temperaturas

A partir dos dados experimentais apresentados na Tabela 1 foram plotados em um gráfico o logaritmo da atividade enzimática da POD (adimensionalizada) em função do tempo, para as diferentes temperaturas em estudo, apresentados na Figura 1. 
Figura 1. Curvas de inativação térmica da POD da água de coco em função da temperatura do tratamento térmico.

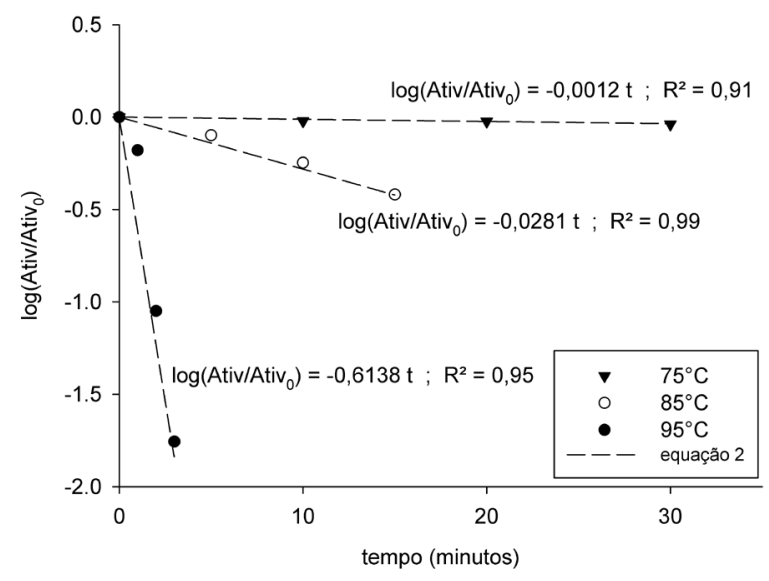

O ajuste do modelo apresentado na equação 2 aos dados experimentais a cada temperatura estudada também é apresentado na Figura 1, bem como os respectivos coeficientes de determinação $\left(\mathrm{R}^{2}\right)$. Por meio das equações ajustadas foram obtidos os valores de $D$ para a inativação térmica da POD nas temperaturas estudadas. Tais valores são apresentados na Tabela 2 .

Tabela 2. Parâmetros cinéticos obtidos para o processo de inativação da peroxidase da água de coco.

\begin{tabular}{cc}
\hline Parâmetro & Valor \\
\hline$D_{75}$ & $833,3 \mathrm{~min}$ \\
$D_{85}$ & $35,6 \mathrm{~min}$ \\
$D_{95}$ & $1,63 \mathrm{~min}$ \\
$Z$ & $7,4^{\circ} \mathrm{C}$ \\
$Q_{10}$ & 22,7 \\
$E_{a}$ & $332,3 \mathrm{~kJ} / \mathrm{mol}$ \\
\hline
\end{tabular}

Como pode ser observado, o valor D a $75^{\circ} \mathrm{C}\left(D_{75}\right)$ foi elevado (833,3 $\mathrm{min})$, sendo esta temperatura ineficiente para o tratamento térmico, uma vez que nessa condição ocorreria o escurecimento do produto antes da enzima ser inativada (CAMPOS et al., 1996).

Para a temperatura de $85^{\circ} \mathrm{C}$ foi obtido um valor $D\left(D_{85}\right)$ de 35,6 min., ainda considerado elevado se assumirmos como eficiente um tratamento que reduza a atividade da POD em $99 \%$ ou dois ciclos logarítmicos $(2 D)$. No entanto, para a redução de $90 \%$ da atividade enzimática, o binômio $85^{\circ} \mathrm{C} / 36$ min. poderia ser facilmente obtido em trocadores de calor menos sofisticados, com menor grau tecnológico requerido, como, por exemplo, tachos abertos encamisados. Contudo, mais estudos e informações sobre a vida de prateleira e as alterações sensoriais da água de coco nessa condição de tratamento são necessários para se verificar sua real aplicabilidade.

E para a temperatura de $95^{\circ} \mathrm{C}$ verificou-se que o valor $D\left(D_{95}\right)$ foi igual a 1,63 min., consideravelmente menor se comparado aos valores de $D_{75}$ e $D_{85}$, podendo ser uma alternativa para a pasteurização rápida da água de coco. Entretanto, Campos et al. (1996) relataram que após 90 segundos de exposição à temperatura de $90^{\circ} \mathrm{C}$, a inativação da POD não é completa e a água de coco começa a apresentar alterações no sabor e aroma, sendo também necessárias mais pesquisas para avaliar tais efeitos provocados pelo tratamento térmico a $95^{\circ} \mathrm{C}$.

Os valores $D$ encontrados para a inativação da POD da água de coco reforçam as informações relatadas por outros autores, no que diz respeito à resistência da POD à inativação térmica (YU et al., 2010; LUÍZ; HIRATA; CLEMENTE, 2007).

Luíz, Hirata e Clemente (2007) estudaram a cinética de inativação da peroxidase em abacate e verificaram que o tratamento a $80^{\circ} \mathrm{C}$ por $10 \mathrm{~min}$. reduziu $34,2 \%$ da atividade da POD. No presente trabalho foi verificado que para um tratamento a $85^{\circ} \mathrm{C}$ por $10 \mathrm{~min}$., a atividade da POD foi reduzida em cerca de $43 \%$. Segundo tais autores, a diferença observada na redução da atividade da POD pode ser atribuída à diferença de composição entre os meios onde a enzima está presente. Os frutos do abacate possuem em média $16 \%$ de lipídeos na composição da sua polpa (TANGO; CARVALHO; SOARES, 2004), valor considerado elevado se comparado ao encontrado na água de coco, cerca de 0,1\% (CAMPOS et al., 1996; CARVALHO et 
al., 2006). O maior teor de lipídeos da polpa de abacate faz com que sua condutividade térmica seja menor, dificultando a transferência de calor nesse meio e conseqüentemente reduzindo a eficiência do tratamento térmico.

Yu et al. (2010) estudaram a cinética de inativação térmica da POD em batata e observaram valores de $D$ na faixa de 57,8 segundos $\left(D_{140}\right)$ a 128,2 segundos $\left(D_{100}\right)$. Tais valores de $D$, considerados elevados para as temperaturas utilizadas, reforçam a termoresistência da POD observada no presente trabalho, demonstrando que são necessárias altas temperaturas, acima de $95^{\circ} \mathrm{C}$, para que se consiga uma inativação eficiente da POD em um curto espaço de tempo.

\section{Constante de resistência térmica (valor $z$ )}

A variação experimental do valor $D$ com a temperatura é uma relação exponencial, linearizada em um gráfico do logaritmo do valor $D$ versus temperatura. Esta curva reflete a resistência relativa da enzima em estudo a diferentes temperaturas.

De posse dos resultados experimentais de $D$ (Tabela 2), foi plotado um gráfico do logaritmo do valor $D$ versus a temperatura, ajustando-se o modelo da equação 3 aos dados experimentais por meio de análise de regressão linear. Os resultados obtidos são apresentados na Figura 2.

A partir da equação apresentada na Figura 20 valor $z$ foi calculado, sendo obtido um valor igual a $7,4^{\circ} \mathrm{C}$ (Tabela 2). Tal valor foi próximo aos reportados por Aguiar, Yamashita e Gut (2008) para a POD termolábil de leite bovino $\left(\mathrm{z}=7,8^{\circ} \mathrm{C}\right)$.

No entanto, valores distintos para $z$ vêm sendo relatado em função das diferentes origens da enzima, variando de cerca de $10,7^{\circ} \mathrm{C}$ a $27,3^{\circ} \mathrm{C}$ (LING; LUND, 1978; ROSENTHAL; RODRIGUES; SLONGO, 2002; MATSUI et al., 2007; GALDINO; CLEMENTE, 2008).
Figura 2. Curva de resistência térmica da POD da água de coco em função da temperatura de inativação térmica.

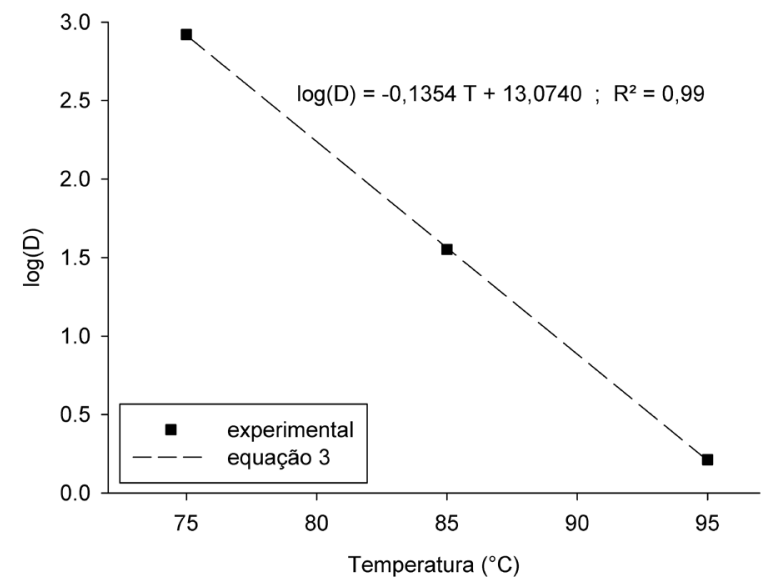

Energia de ativação (E) e Coeficiente de temperatura $\left(Q_{10}\right)$

Uma vez determinados os valores $D$ para as temperaturas em estudo, as constantes de velocidade da redução da atividade enzimática $(k)$ podem ser calculadas utilizando-se a Equação 6 . Foram plotados então em um gráfico os valores do logaritmo natural de $\mathrm{k}(\operatorname{lnk})$ em função do recíproco da temperatura $(1 / \mathrm{T})$, para avaliar o ajuste do modelo linearizado de Arrhenius (Equação 5) aos dados experimentais. Tais informações são apresentadas na Figura 3.

Figura 3. $\ln (k)$ em função de $1 / T$, para determinação da energia de ativação do processo de inativação térmica da POD da água de coco.

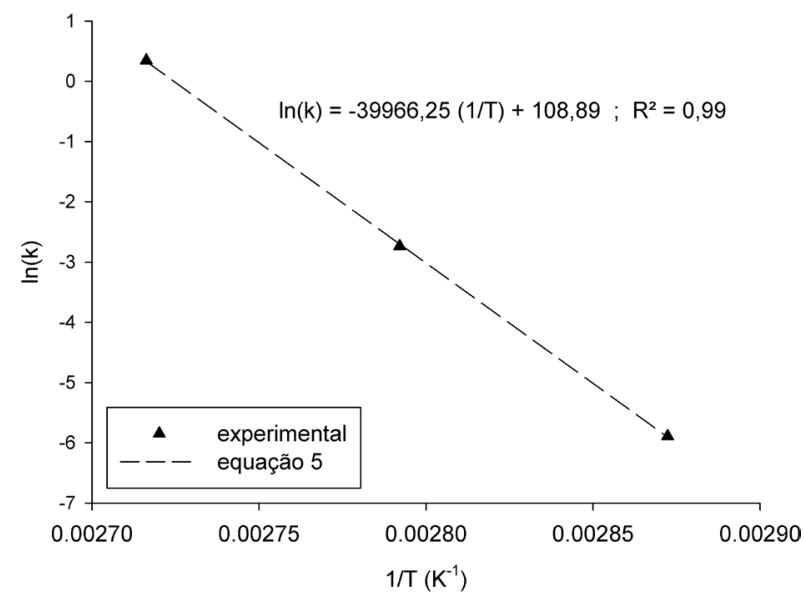


A partir da equação ajustada, verificou-se que a energia de ativação $\left(E_{a}\right)$ para o processo de inativação térmica da POD da água de coco foi de $332,3 \mathrm{~kJ} / \mathrm{mol}$ (Tabela 2), valor considerado elevado e que reforça a característica de resistência da POD a tratamentos térmicos, sendo requerida grande quantidade de energia em seu processo de inativação.

Uma vez conhecido o valor da $E_{a}$, a equação 4 foi utilizada para calcular o valor do coeficiente de temperatura $\left(Q_{10}\right)$ no intervalo estudado. Verificouse que o valor médio para $Q_{10}$ foi de 22,7 , mostrando que o processo de inativação térmica da POD é fortemente afetado pela temperatura escolhida para o tratamento térmico

A POD apresentou-se como um bom indicador da eficiência de um tratamento térmico, apresentando grande resistência quando comparado a outras enzimas e micro-organismos. Uma vez determinados tais parâmetros cinéticos, novos binômios tempo/temperatura poderão ser propostos para o tratamento térmico da água de coco, sendo necessários mais estudos a fim de se verificar o efeito nas características físico-químicas e sensoriais da mesma, para que seja obtido um produto final de qualidade e aceitável pelo consumidor.

\section{Conclusões}

A inativação térmica da enzima POD presente na água de coco foi estudada, verificando-se o efeito da temperatura utilizada, na faixa de $75^{\circ} \mathrm{C}$ a $95^{\circ} \mathrm{C}$, sendo encontrados os valores $D$ entre $833 \mathrm{~min}$ (a $75^{\circ} \mathrm{C}$ ) e $1,6 \min \left(\mathrm{a} 95^{\circ} \mathrm{C}\right)$ e um valor $z$ igual a $7,4^{\circ} \mathrm{C}$. O valor de $Q_{10}$ obtido, 22,7, demonstra que a cinética de inativação enzimática foi fortemente afetada pelo aumento da temperatura, e o valor de $332,3 \mathrm{~kJ} / \mathrm{mol}$ encontrado para a energia de ativação do processo indica que a POD da água de coco tem grande resistência à inativação, sendo um bom indicador da eficiência do tratamento térmico aplicado.

\section{Agradecimentos}

À UESB pelo suporte técnico e profissional.

\section{Referências}

AGUIAR, H. F.; YAMASHITA, A. S.; GUT, J. A. W. Estudo de indicadores enzimáticos de determinação rápida para avaliação da pasteurização contínua. In: CONGRESSO BRASILEIRO DE CIÊNCIA E TECNOLOGIA DE ALIMENTOS, 21., 2008, Belo Horizonte. Anais... Belo Horizonte: Sociedade Brasileira de Ciência e Tecnologia de Alimentos, 2008. p. 1-3.

BALL, C. O.; OLSON, F. C. W. Sterilization in food technology. New York: McGraw-Hill, 1957.

CAMPOS, C. F.; SOUZA, P. E. A.; COELHO, J. V.; GLÓRIA, M. M. B. A. Green coconut water quality. Journal of Food Processing and Preservation, New Jersey, v. 20, n. 6, p. 487-500, 1996.

CARVALHO, J. M.; MAIA, G. A.; SOUSA, P. H. M.; MAIA JUNIOR, G. A. Água-de-coco: propriedades nutricionais, funcionais e processamento. Semina: Ciências Agrárias, Londrina, v. 27, n. 3, p. 437-452, 2006.

DUARTE, A. C. P.; COELhO, M. A. S.; LEITE, S. G. F. Identification of peroxidase and tyrosinase in green coconut water. Ciencia y Tecnologia Alimentaria, Espanha, v. 3, n. 5, p. 266- 270, 2002.

EL-LOLY, M. M.; AWAD, A. A.; MANSOUR, A. I. A. Thermal kinetics denaturation of buffalo milk immunoglobulins. International Journal of Dairy Science, Faisalabad, v. 2, n. 4, p. 292-301, 2007.

FELLOWS, P. J. Tecnologia do processamento de alimentos. 2. ed. Porto Alegre: Artmed, 2006. 602 p.

FREITAS, A. A.; FRANCELIN, M. F.; HIRATA, G. F.; CLEMENTE, E.; SCHMIDT, F. L. Atividades das enzimas peroxidase (POD) e polifenoloxidase (PPO) nas uvas das cultivares benitaka e rubi e em seus sucos e geléias. Ciência e Tecnologia de Alimentos, Campinas, v. 28, n. 1, p. 172-177, 2008.

GALDINO, N. O.; CLEMENTE, E. Palmito de pupunha (Bactris gasipaes Kunth.): composição mineral e cinética de enzimas oxidativas. Ciência e Tecnologia de Alimentos, Campinas, v. 28, n. 3, p. 540-544, 2008.

LING, A.; LUND, D. Determining kinetic parameter for thermal inactivation of heat-resistant and heat-labile isozymes from thermal destruction curves. Journal of Food Science, Chicago, v. 43, n. 4, p. 1307-1310, 1978. 
LUÍZ, R. C.; HIRATA, T. A. M.; CLEMENTE, E. Cinética de inativação da polifenoloxidase e peroxidase de abacate (Persea americana Mill.). Ciência e Agrotecnologia, Lavras, v. 31, n. 6, p. 1766-1773, 2007.

MAGALHÃES, M. P.; GOMES, F. S.; MODESTA, R. C. D.; MATTA, V. M.; CABRAL, L. M. C. Conservação de água de coco verde por filtração com membrana. Ciência e Tecnologia de Alimentos, Campinas, v. 25, n. 1, p. $72-$ 77, 2005.

MATSUI, K. N.; GRANADO, L. M.; OLIVEIRA, P. V.; TADINI, C. C. Peroxidase and polyphenol oxidase thermal inactivation by microwaves in green coconut water simulated solutions. $L W T$, New York, v. 40, n. 5, p. 852-859, 2007.

PENNA, T. C. V.; MACHOSHVILI, I. A. Esterilização térmica. conceitos básicos da cinética de morte microbiana. Revista Farmácia Bioquímica USP, São Paulo, v. 34, p. 1-5, 1997. Suplemento 1.

ROBINSON, D. S.; ESKIN, N. A. M. Oxidative enzymes in foods. New York: Elsevier Applied Science, 1991.
ROSENTHAL, A.; RODRIGUES, A. M.; SLONGO, A. P. Cinética de inativação térmica da peroxidase de suco de manga. In: CONGRESSO BRASILEIRO DE FRUTICULTURA, 17., 2002, Belém. Anais... Belém: Sociedade Brasileira de Fruticultura, 2002. CD-ROM.

TANGO, J. S.; CARVALHO, C. R. L.; SOARES, N. B. Caracterização física e química de frutos de abacate visando a seu potencial para extração de óleo. Revista Brasileira de Fruticultura, Jaboticabal, v. 26, n. 1, p. 1723, 2004.

YU, B.; JIN, Z.; DENG, L.; XU, X.; HE, L.; WANG, J.; TIAN, Y.; CHEN, H. Kinetic study of thermal inactivation of potato peroxidase during high-temperature short-time processing. Journal of Food Science and Technology, New Delhi, v. 47, n. 1, p. 67-72, 2010.

ZANATTA, C. L.; ZOTARELLI, M. F.; CLEMENTE, E. Peroxidase (POD) e polifenoloxidase (PPO) em polpa de goiaba (Psidium guajava R.). Ciência e Tecnologia de Alimentos, Campinas, v. 26, n. 3, p. 705-708, 2006. 
